\title{
I pionieri italiani della geofisica $(*)$
}

\author{
P. CALOI
}

Ricevuto il 18 Maggio 1963

Riassunto. - Vengono rapilamente passati in rassenga, seguendo l'ordine cronologico, gli studiosi di geofisica - intesa in senso lato - che, a partire dalla seconda meta del Settecento, ebbero ad interessarsi in Italia di elettricita atmosferica, magnetismo terrestre, idrografia, oceanografia fisica, sismologia e vulcanologia, con intenti pionieristici. Sono ricordati i principali risultati raggiunti da una trentina di ricercatori fra i quali spiceano, per la vastita del lavoro compinto e per la novita dei risultati, Leopoldo Nobili e Macedonio Melloni. Ma, ciascuno nel suo campo, scoperte di rilievo fecero pure G. B. Beccaria, Luigi Palmieri, Carlo Matteucci, Timoteo Bertelli, Luigi De Marchi, Carlo Somigliana, Emilio Oddlone, Alfonso Sella e Iomenico Pacini. Aleni vantano priorita di rilievo: scoperta della doppia oscillazione diurna del barometro (Chiminello, 1780); unicita sorgiva delle varie radiazioni dello spettro (M. Melloni); sismografo elettromagnetico a registrazione continua (Palmieri, 1855); microsismi (Bertelli, 1872); il paleomagnetismo (Oddone e Sella, 1891); la radiazione cosmica (Pacini, 1910); ...

Summary. - The paper briefly recalls, in ehronological order, the scientists who, after the XVII century, took interest in geophysics - in a broad sense of the word - and especially in the sectors of atmospheric electricity, seismology and vulcanology, earth magnetism, hydrography, physical oceanography, acting as pioneers in these fields. The principal results achieved by some 30 research workers, among whom a particularly wide and new work was performed by Leopoldo Nobili and Macedonio Melloni, are recalled. Each in his own field, also G. B. Beccaria, Luigi Palmieri, Carlo Mattenci, Timoteo Bertelli, Luigi De Marchi Carlo Somigliana, Emilio Oddone, Alfonso Sella e Domenico Pacini achieved very remarkable discoveries.

(*) La traduzione in lingua inglese e apparsa nel volume VII - 2 dei "Cahiers d'Histoire Mondiale", pubblicati sotto gli auspici dell'UNESCO nel 1963. 
$\Lambda$ few hoast important priorities: discovery of the double daily oscillation of barometres (Chiminello, 1780); sigle source of the various spectrum radiations (M. Melloni); continuous recording electromagnetic seismograph (Palmieri, 1855); microseism (Bertelli, 1872), paleomagnetism (Oddone and Sella, 1891); cosmic radiation (Pacini, 1910): etc.

1. - Il termine "pionieri " richiama alla mente quegli esploratori che, per primi, si inoltrarono in vaste regioni sconosciute o, comunque, poco note. Ed e un richiamo appropriato, che i due ordini di attivita hanno parecchie caratteristiche comuni. Anelito alla conoscenza di cose nuove, entusiasmo che sopporta ogni forma di rinuncia, disinteresse per l'immediato sfruttanento ai fini materiali, senso del mistero che affascina e sgomenta ad un tempo.

Non e facile orientarsi in estese regioni sconosciute e non tutti i pionieri hanno ugualmente sviluppato il senso dell'orientamento. Inevitabili quindi squilibri di risultati, disparità di giudizi, precarieta di interpretazioni: cammini che sembravano all'inizio ricchi di prospettive, si rivelano improvvisamente vicoli ciechi; sentieri aridi e dirupati, che sfociano alla fine in zone caratterizzate da insospettata abbondanza di speranze e di certezze.

Caratteristica comune delle acquisizioni dei pionieri (in senso lato) e l'incertezza dei contorni: prospettive, spesso possenti, ma appena rozzamente delineate, dai confini indecisi. D'altronde, l'esploratore che si apre a colpi d'accetta la via nella foresta, non può preoccuparsi di renderla agevole, comoda, ben definita: questo sara compito di altri. Le rifmiture, le delimitazioni precise non è opera di pionieri.

Va ancora osservato che non sempre il pioniere e facilmente individuabile. Non e infrequente il caso di esploratori, le scoperte dei quali restano ignorate per secoli; o vengono attribuite ad altri. Questi sono casi estremi, e vero. Pur prescindendo da essi, sovente un ricercatore assume la figura del pioniere solo perche ha espresso in forma piu comprensibile, ed ha saputo abilmente diffondere in vasta cerchia, scoperte nettamente delineate nell'opera di un precedente ricercatore, al quale va soltanto la qualifica di pioniere.

Desidero accennare ad un altro aspetto del pionierismo: all'influsso cioe che, sulla notorieta di un pioniere, puo determinare il chiamarsi in un modo anziche in un altro. Alighieri, Galilei, Volta, ... sono nomi troppo profondamente accaparrati, perche ricercatori omonimi non restino diminuiti nella loro notorieta. Cio vale, naturalmente, in senso generale, e quindi anche per i pionieri. A questo riguardo, con mia 
sorpresa, ho trovato fra i pionieri della geofisica un Becearia, un Garibaldi e un Sella. Devo confessare che, specie i primi due, mi risultavano del tutto nuovi come pionieri della geofisica. Il portare quei nomi gia tanto celebri, in altre attivita dello scibile, indipendentemente dai meriti, li ha indubbiamente molto svantaggiati.

Dopo questa premessa, sufficiente del resto a mostrare quanto sia arduo il compito di riuscire imparziali nella cernita e nella giusta valutazione dei pionieri, entro senz'altro nell'ampio, fiorito giardino e cerco di scernere fior da fiore.

2. - Ritengo superfluo risalire troppo addietro nel tempo in questa mia cernita. Nel XVIII secolo troviamo p. es. un monaco, certo BIRRA, che nel 1751 costruì un pendolo verticale, con penna scrivente. Ma sia questi, che il Conte nelLA ToRRe (1795) ed altri dell'epoca, non portarono veri contributi scientifici, limitandosi a mere, grossolane osservazioni di terremoti, intraducibili in campo scientifico.

La prima personalità spiccata clee, nell'ambito degli studi geofisici, troviamo nel XVIII secolo e G. B. BEccirIA (Mondovi 1716 - Torino 1781). Si interessò particolarmente di meteorologia, elettricita atmosferica e magnetismo terrestre. Usò, fra i primi, barometri scriventi. Si propose la determinazione delle leggi delle variazioni regolari dell'elettricita atmosferica durante il giorno, le sue relazioni con i fenomeni meteorici, ece. Determino l'esistenza di scariche convettive fra la terra e l'atmosfera. Introdusse in Piemonte l'uso del parafulmine, cercando di perfezionarlo. Si avvide per primo dell'elettrolisi. Dalle sue esperienze fu condotto ad affermare che la causa del magnetismo terrestre e una circolazione regrolare e costante di elettricita da nord a sud. Nelle sue "Lettere sull'elettricismo ", adottò l'opinione dell'astronomo ed idraulico PAoLo Frisi (1727-1814), essere l'aurora boreale dovuta a scariche elettriche prodotte nelle regioni elevate dell'atmosfera, affermazione veramente notevole per l'epoca. Fondamento di questa ipotesi erano: le apparenze luminose che, per primo, egli aveva osservato nel vuoto al passaggio dell'elettricita; il fatto che l'aria diviene di piu in piu rarefatta a mano a mano che aumenta la quota; le osservazioni di Halley, che collegò l'aurora boreale al magnetismo terrestre; il concetto enunciato dallo stesso Beccaria circa l'identita fra i fenomeni magnetici e quelli elettrici.

Del settecento possiamo citare ancora GIUseppe Toaldo (17191798) e Vincenzo Chiminello (1741-1815) che iniziarono, a Padova, regolari registrazioni di osservazioni barometriche. Con essi, per la prima volta, si tenta di rintracciare delle periodicita nell'andamento della pressione atmosferica. Cosi, mentre Toaldo credette di scopriere, nei fenomeni 
meteorologici, una periodicita di 18 mesi (ciclo toaldino), Chiminello (1780) ritenne di aver constatata l'esistenza di due massimi e di due minimi barometrici diurni, il che costituisce un'autentica priorita.

Nel primo ottocento merita menzione Francesco CarLinI (17831862), che si interessó di meteorologia e, in particolare, delle variazioni della pressione. Egli peró va ricordato sopra tutto per aver ideato un sistema di determinazione della densita della Terra, basato sul numero delle oscillazioni che un pendolo compie separatamente sulla cima di una montagna e in pianura. Carlini applicò (1824) tale metodo sul Moncenisio ed ottenne per la densita della Terra il valore 4,84, che si discosta per difetto di 0,7 circa da quello oggi accettato.

La prima meta del XIX secolo e peró dominata nel campo della fisica e, in parte, della geofisica, da due nomi celebri: Leopoldo Nobili e Macedonio Melloni.

L. NobILI (1784-1835) patriota modenese, fisico illustre. Della sua opera vasta, accenneremo solo a quella parte che può avere attinenza con la geofisica.

Com'e noto, Ampere provò che il magnetismo puo essere considerato come conseguenza di fenomeni elettrici. Nella teoria di Ampere, le molecole di una calamita sono percorse da tenui correnti circolari, tutte dirette nello stesso senso, perpendicolarmente all'asse del magnete. Ampere aveva applicato la sua teoria al magnetismo terrestre, ritenendo quest'ultimo come conseguenza delle correnti elettriche, da cui la Terra, considerata come sfera conduttrice, è percorsa sensibilmente in direzione da est a ovest. Leopoldo Nobili realizzò sperimentalmente l'ipotesi di Ampere, stendendo lungo i paralleli di una sfera di legno un filo continuo di rame isolato: facendolo percorrere da una corrente, egli riprodusse in dettaglio le caratteristiche dell'azione della supposta Terra sull'ago magnetico. Tale esperienza e impropriamente attribuita al fisico inglese Barlow che riprodusse a Londra lo stesso apparecchio due anni dopo Nobili.

Ma il Nobili merita di essere citato come l'ideatore di apparecchi, atti a rivelare le minime correnti elettriche. Dopo la scoperta di Oersted, spetta a Schweigger la costruzione di uno strumento, detto allora moltiplicatore, col quale avvolgendo ripetutamente un filo percorso da corrente intorno ad un ago magnetico, fu possibile scoprire l'esistenza di correnti debolissime moltiplicandone, per cosi dire, l'azione; correnti tanto deboli da influenzare in modo insufficiente l'ago magnetico nella semplice esperienza di Oersted. Nel 1835, Nobili ebbe il merito di perfezionare tale apparecchio, applicandovi il sistema astatico e, successivamente, 
sospendendo il sistema ad un filo di bozzolo, tutto opportunamente protetto da una campana di vetro. Di qui ebbe origine il galvanometro, strumento preziosissimo in molte branche della Geofisica. Originariamente, nel galvanometro di Nobili, allo scopo di sottrarre lo strumento all'azione del campo magnetico terrestre, due aghi astatici paralleli, perfettamente uguali in dimensione e in forza magnetica, venivano immessi nello stesso filo, in direzione contrarie. Con tale strumento, Nobili studio numerosi fenomeni fisiologici, in precedenza ignorati, e scoprì le correnti idro-elettriche.

La multiforme attivita di Nobili si rese benemerita anche in altri campi della fisica. Ai primordi dell'Ottocento iniziarono gli studi sul calore raggiante, sopratutto per merito di Rumford e di Leslie; studi che condussero poi al concetto di potere emissivo. Gia il Leslie e il Rumford avevano ideato dei termometri differenziali, atti allo studio di questi fenomeni. Spetto pero a Nobili il conseguimento di un decisivo progresso nell'efficacia di questi apparecchi: nel 1829, egli infatti costruì il termomoltiplicatore, che doveva servire con vantaggio in tutte le successive ricerche relative al calore raggiante. Di esso si valse efficacemente anche Macedonio Melloni.

Il termo-moltiplicatore di Nobili e una versione notevolmente perfezionata dell'apparechio di Seebeck: egli moltiplica gli elementi bismuto ed antimonio, li unisce fra loro alternativamente ad angolo sempre piu acuto, formando, in tal moto - per evidente analogia con la pila voltaica - una vera e propria pila termo-elettrica, opportunamente raccolta in forma di cilindro metallico, allo scopo di raccogliere le minime quantita di raggi calorifici, provenienti dai corpi circostanti.

Insieme con Vincenzo Antinori (1792-1865), Nobili ottenne, fra l'altro, le correnti indotte dal magnetismo terrestre in una spirale mossa nel meridiano magnetico.

L'altro eminente scienziato, di fama europea, che ha onorato l'Italia, nel campo che ci interessa, nella prima meta dell'Ottocento fu MACEDonio Melloni (1798-1854). Fisico illustre, uno dei massimi che l'Italia abbia avuto dopo Volta, nacque a Parma ed ebbe vita agitata, per le sue idee politiche, che lo portavano a combattere ogni forma di timannide. Inizio il suo insegnamento all'Universita di Parma nel 1824. Nel 1830 dovette emigrare a Parigi, per avere apertamente lodato gli studenti parigini che presero parte alla rivoluzione del 1830. Dopo varie vicende, in seguito all'interessamento di Arago e di Humboldt, divenne professore a Napoli, dove mori di colera nel 1854, dopo aver perduto l'insegnamento per la seconda volta, a motivo delle sue idee liberali. 
Molte delle sue ricerche hanno attinenza con la Geofisica.

Di particolare interesse sono quelle sul calore raggiante.

Cominciò col perfezionare il termo-moltiplicatore di Nobili: per la sua termo-pila si valse del bismuto e dell'antimonio (particolarmente sensibili all'eccitamento dell'azione termo-elettrica), ma ne rese gli elementi perfettamente identici alle due stremita, piu sottili e piu numerosi. La rese inoltre indipendente dalle variazioni della temperatura dell'aria e dalle perturbazioni legate al calore proprio dei corpi circostanti. Ne aumentò la prontezza e la rese di estrema sensibilita. Con questo apparecchio, Macedonio Melloni compi una lunga serie di ricerche, con risultati di eccezionale interesse. Con un prisma di salgemma dimostró la rifrazione dei raggi calorifici piu oscuri e con una lente di salgemma riusci a concentrarli. Provo la polarizzazione dei raggi termici, la loro propagazione rettilinea, istantanea ed indipendente dalle fluttuazioni dell'aria. Dimostro che l'intensita d'irraggiamento decresce con il quadrato della distanza. Trovo le leggi fondamentali della diffusione ed emissione calorifica; come si verifica l'abbassamento di temperatura per irraggiamento, spiegando compiutamente anche il fenomeno della rugiada. Dimostro la varia rifrangibilita dei raggi termici e dei raggi chimici (attinici); il diverso potere selettivo dei corpi per gli uni e per gli altri, analogamente al comportamento dei mezzi trasparenti colorati, nei riguardi delle radiazioni luminose (termocrosi); pervenne all'analisi calorifica dello spettro solare. A questo proposito, egli riconobbe che la posizione del massimo di calore nello spettro dato da un prisma varia con la sostanza di cui il prisma e formato - perche, le varie sostanze assorbono in modo diverso i raggi termici - e che un prisma di salgemma, diafano e limpido, dava lo spettro calorifico normale, perche quasi non assorbe il calore (solo lo 0,08 del calore incidente, come egli determino). Scoprì inoltre che l'aria secca e perfettamente trasparente ai raggi calorifici.

Ma uno dei massimi meriti di Melloni fu di aver dimostrato che i fenomeni luminosi e i fenomeni calorifici derivano da un'unica serie di radiazioni, di cui una parte agisce sull'organo della vista e l'altra si svela ai nostri sensi per il riscaldamento dei corpi. Provò, in altri termini, l'unicità sorgiva delle varie radiazioni dello spettro, chimiche, luminose, alorifiche, contro l'opinione di Leslie e della maggior parte dei fisici di allora. Dimostrò non esservi luce senza calore.

Per quanto afferisce strettamente alla geofisica, oltre ad alcune ricerche di meteorologia (variazioni della pressione atmosferica e venti ad essa collegati) va citata anche l'invenzione del magnctoscopio, con il 
quale egli seoprì nuove verita, così da dare un nuovo indirizzo agli studi sul magnetismo terrestre. Fra l'altro, esamino col nuovo strumento gran copia di minerali e provo che la doppia polarita magnetica - trovata dal prof. Scacehi per alcuni frammenti di lava rinvenuti sul Vulture - e comune a tutte le lave a quasi tutte le roce. Studio, a questo riguardo, le lave del Vesuvio e $\mathrm{i}$ vari aspetti della bipolarita delle rocee.

Costruì infine un nuovo, sensibilissimo elettroscopio, con il quale si riprometteva lo studio dell'elettricita di tensione, proposito troncato dalla morte prematura.

Te sue esperienze furono in parte laccolte nell'opera "La Thermochrose ou la coloration calorifique ", pubblicata nel 1850, quattro anni prima della sua morte.

Nella prima meta dell'Ottocento troviamo ancora LitgI MAGRIs] (1802-1868). Si laureo a Padova ingegnere architetto nel 1825. Dopo varie vicende, nel 1863 fu nominato professore di fisica all'Istituto superiore di perfezionamento di Firenze. Precedette Steinheil e Wheatstona nella realizzazione del telegrafo (1837). Si interesso molto di questioni relative alle correnti elettriche, con innegabili successi. I dimitandoci al suo interesse per questioni geofisiche, diremo che volse i suoi studi sopla tutto all'elettricita atmosferica e alla meteorologia. Sulla base degli effetti del fulmine, fu portato a studiare la costruzione dei parafulmini. Egli trovò che un conduttore, interrotto da condensatore munito di spinterometro a punte, aumenta il trasferimento dell'elettricita dalle nubi al suolo.

Insieme a Francesco Carlini, nel 1861, progetto la fondazione della Societa Meteorologica italiana. Si interesso di correnti telluriche, studio numerose trombe d'aria, elaboro le osservazioni meteorologiche fatte in Udine nel quarantennio 1803-1842 (1852), si oceupo degli effetti prodotti dalle nubi temporalesche sulle linee telegrafiche (1852-1854), fece alcune indagini sulla formazione della grandine, sulla durata della striscia luminosa lungo il ammino percorso dal fulmine (1861): raccolse parte delle sue scoperte nella pubblicazione "Sull'elettricita atmosferica " (1863).

Iuigr Palmieri (1807-1896). Nacque a Faichio (Benerento) e molì a Napoli. Studio matematica, fisica e filosofia. Nel $18+7$ sostitui Galluppi nell'insegnamento della filosofia nell'Universita di Napoli. Nel 1856 divenne direttore dell'Osservatorio vesuriano. Nel 1860 , ceduta la cattedra di filosofia a Bertrando Sparenta, passo a quella di fisica terrestre, espressamente istituita per lui. Come fisico, le sue ricerche si riferiscono quasi esclusivamente allo studio delle caratteristiche della Terra, Nel $\mathbf{1 8 4 0}$ 
pubblicò una memoria dal titolo: "Alcune esperienze del magnetismo terrestre ed invenzione di una batteria magneto-elettrica-tellurica ". Questo lavoro e alla base di tutta una serie di ricerche di geofisica. E stato il primo ad ottenere la scintilla e la scossa delle correnti indotte direttamente dal magnetismo terrestre; inoltre, i suoi studi di elettricita atmosferica possono ritenersi iniziatori degli sviluppi di questo ramo della meteorologia. Con il suo elettrometro bifilare aprì la strada alla ricerca delle leggi e dell'origrine dell'elettricita atmosferica. Investigò il comportamento dell'elettricita anche nel mezzo solido. Uno dei suoi ultimi lavori verte appunto su "Le correnti telluriche all'Osservatorio vesuviano osservate con fili inclinati all'orizzonte durante l'anno 1895 ".

Si interessó pure di sismologia e di vulcanologia. Nel 1852 descrive e ricerca le cause del terremoto avvenuto nella zona del Vulture.

Successivamente, descrive le eruzioni del Vesuvio avvenute nel 1861 e nel 1872. Ideó pure un sismografo elettromagnetico (1855), che puo ritenersi il primo apparecchio sismico a registrazione continua. Sviluppo teorie - oggi naturalmente insostenibili — sulle maree della parte interna fluida della Terra e sulle loro relazioni col vulcanismo. Ottenne risultati notevoli nello studio delle fumarole e delle lave, condotto con metodi chimici e spettroscopici: scopri nelle sublimazioni vesuviane del litio, del talio, dell'antimonio, dell'acido borico, parecehi carbonati alcalini, e principalmente - per la prima volta - riconobbe in una sublimazione del 1881 la caratteristica riga spettrale dell'elio.

Pubblicò quattro volumi degli "Annali dell'Osservatorio Vesuviano ", la "Geologia del Monte Vulture e terremoto quivi avvenuto il 14 Agosto 1851 ", e, pochi mesi prima della morte, "Il Vesuvio dal 1875 al 1895 ".

Studioso di scienze marittime fu Almssandro Cialdi (Civitavecchia 1807 - Roma 1882). Studio alla scuola navale di Genova, prese servizio nella marina sarda e, successivamente, fu comandante di quella pontificia. Viaggiò lungo il Nilo, fino alla sua prima cateratta; passo dall'Italia all'Inghilterra, attraverso fiumi e canali francesi. Fece pregevoli ricerche teoriche e pratiche su costruzioni e canalizzazioni marittime, esposte al moto ondoso e alle correnti. Tali ricerche egli applico, fra l'altro, alla costruzione di Porto-Said.

Fra le sue opere vanno ricordate: "Sul moto ondoso del mare e sulle correnti di esso" (1865), tuttora citato nei trattati di oceanografia, e "Nozioni preliminari per un trattato nella costruzione dei porti nel Mediterraneo " (1874).

Carlo Matteucor (1811-1868). Nacque a Forli, segui gli studi di matematica e fisica all'Universita di Bologna, morì a Pisa. Si fece no- 
tare non solo come valente cultore di scienze, ma anche come nomo politico; fu, fra l'altro, presidente della Societa Italiana dei XL e ministro della pubblica istruzione nel ministero Rattazzi.

Non e mio compito parlare della sua opera di fisico, vasta e complessa. Egli si interesso sopra tutto di elettricita, con particolare riguardo alla elettricita animale e ai fencmeni fisico-chimici dei corpi viventi. Parechi dei suoi lavori hanno chiari riferimenti alla geofisica. A soli 16 anni, mentre frequentava il $2^{\circ}$ anno di universita, pubblico una memoria dal titolo "Sulla influenza dell'elettricita nella formazione delle principali meteore acquee ". Piu tardi, nel 1845 , parallelamente ai suoi lavori di elettro-fisiologia, esegui numerose esperienze intese a verificare se la Terra poteva servire da conduttore della corrente. La sua attenzione fu a lungo attratta dal magnetismo di rotazione e dai sistemi di correnti indotte nel disco. Ma ritorno spesso su fenomeni prettamente geofisici. Fra l'altro, egli si occupo per primo delle perturbazioni elettriche eccitate dalle aurore boreali nei fili telegrafici (1859). Erano gia state eseguite esperienze, intese a scoprire l'esistenza di correnti proprie della Terra. Matteucei fece, a questo riguardo, fondamentali indagini nella pianura di San Maurizio, con due fili conduttori lunghi $6 \mathrm{~km} \mathrm{e}$ ben isolati dal suolo.

Egli trovo che, in assenza di temporali nelle vicinanze, e con aria tranquilla, nello stato normale ordinario, non si aveva corrente - oppure lievissima e variabile in direzione - sul filo normale al meridiano terrestre; mentre nell'altro filo, in direzione del meridiano del luogo, era sempre presente una corrente dal sud al nord, di intensita variabile e con due massimi in due diverse ore del giorno (1865). Non si esauri in queste esperienze, l'interessamento di Carlo Matteucei per questioni di elettricita atmosferica e di magnetismo terrestre.

Tn fisico singolare, Giovanni Cantoni (1818-1897), che si impegno a fondo nel proposito di ricercare i rapporti fra fisica e filosofia, non trascuro di occuparsi, fino dagli anni giovanili, dello studio paziente della meteorologia, con particolare riguardo al fenomeno della rugiada, ai legami fra meteorologia e agraria, agli evaporimetri e alla temperatura dell'aria. Per l'originalita dei suoi studi in questo ramo della scienza, fu nominato direttore del servizio centrale di meteorologia, carica che tenne fino al 1878 e alla quale dedico gran parte della sua attivita.

ANgelo SecchI (Reggio Emilia 1818 - Roma 1878) fu sopratutto astronomo. Ingegno versatile, si interesso altresì di meteorologia ed idrologia, fisica del mare e dell'atmosfera, climatologia e previsione del tempo. Costrui un meteorografo ed un attinometro. 
Egli misuro la trasparenza dell'acqua basandosi sulla visione di un disco metallico immerso ed indicando la profondita alla quale detta visione cessa (dischi Secchi). Con tale mezzo egli compì accurate misure di trasparenza in un viaggio sul Tirreno, a bordo della nave "Immacolata concezione" (1865). In tali misure, oltre a fattori personali, influiscono sulla distanza della visione, la forma, le dimensioni e il colore del disco, nonche le mutevoli condizioni del Sole, del cielo e della superficie dell'acqua. Cio malgrado, Vercelli dimostrò che le indicazioni ottenute con il disco Secchi hanno valore scientifico maggiore di quanto, a prima vista, si potrebbe pensare. Negli ultimi decenni, l'uso del disco Secchi e stato via via sostituito da speciali fotometri per graduare la trasparenza degli strati interni, usando sorgenti di luce artificiale.

Fra le opere di Padre Secchi, ritengo degno di menzione il poderoso lavoro "L'unita delle forze fisiche - Saggio di flosofia naturale " (1864), specie di summa sulle cognizioni fisico-matematiche dell'epoca, riceo di geniali divinazioni e di una appassionata fiducia nell'avvenire della scienza.

Un altro fisico che si occupo di questioni di geofisica fu Alessaxdro SERPIERI (1823-1885); trattò argomenti di meteorologia, astronomia, sismologia e fisica terrestre. Fondo l'osservatorio meteorologico di Urbino, presso la cui Universita fu professore di fisica.

Una citazione speciale merita Pier Maria GardBaldi (Chiavari 1823 - Genova 1902). Laureatosi nel 1850 in medicina, si dedicò ben presto agli studi sperimentali, collaborando con M. A. Bancalari, professore di fisica all'Universita di Genova, in molte ricerche sull'elettromagnetismo. In modo particolare, si interessò del diamagnetismo dei gas.

Accanto alle sue ricerche prettamente fisiche, condusse cospicui lavori nel campo della fisica terrestre. Contribui validamente alla conoscenza della climatologia ligure, di Genova in particolare, determinando i valori normali degli elementi climatologici, tratti da un periodo di 60 anni di diligenti osservazioni (1895).

La maggiore attenzione egli riservo ai problemi di magnetismo terrestre. A conclusione di una lunga serie di osservazioni e di studi, nel 1884 egli pote stabilire che "le perturbazioni o burrasche magnetiche sono regolate dalla stessa legge fisica, che governa le variazioni dell'ago magnetico e che, come queste, hanno la loro misura nel numero e nell'ampiezza delle macchie solari, e che si svolgono in egual periodon.

Egli studiò le relazioni fra le macchie solari e il magnetismo terrestre durante l'ultimo trentennio del secolo XIX. 
Nel 1870 fu nominato direttore dell'Osservatorio meteorologico di Genova.

Nel campo dei micromovimenti della crosta terrestre Trmoteo Bertell (1826-1905) e pioniere indiscusso. Entro nel 1844 nell'ordine dei Barnabiti. Passo in diverse case di questo ordine (Moncalieri, Napoli, ecc.), finche giunse al Collegio alle Querce (Firenze) nel 1868. Fu qui che egli pote studiare i piccoli spontanei movimenti del pendolo. Dopo alcuni anni di pazienti osservazioni (basti ricordare che - almeno per i primi tre anni - egli osservava con una lente i movimenti dell'estremita di un appendice di una massa pendolare, sospesa ad un robusto braccio infisso in una parete) egli pote suggerire quattro cause per i movimenti pendolari: l'azione del vento, le correnti convettive nella custodia dello strumento, le vibmazioni legate al passaggio dei veicoli, le espansioni a contrazioni dell'edificio sotto i cambiamenti di temperatura. Il merito eccezionale delle ricerche di Bertelli sta pero nell'aver indicato, nel Novembre 1872 , fra le cause di perturbazione pendolari il passaggio delle depressioni barometriche. Con ciò egli scopriva la causa principale a cui sono legati i cosi detti microsismi. Esatta si dimostrava anche l'altra sua conclusione: rivelare cioe i microsismi un massimo invernale ed un minimo estivo.

Nell'ambito della scienza idrografica non puo essere ignorato il nome di Giovanni Battista Magnaghi, ammiraglio idrogtafo (Lomello 1839 - Roma 1902). A lui si deve l'organizzazione e il rapido sriluppo dell'Ufficio idrografico della R. Marina, istituito a Genova nel 1872. Diede potente impulso al rilievo idrografico delle coste italiane e delle colonie, affrancando l'Italia dal ricorso a carte straniere. Farori la talassografia e organizzo numerose campagne di rilevamento e di scandaglio.

Giuseppe Basso (Chivasso 1842 - Torino 1895) fu fisico molto noto: si interessò di questioni di magnetismo, di acustica e, in modo preminente, di ottica fisica. Solo sporadicamente la sua attenzione fu attirata da fenomeni geofisici. Ricorderemo, a questo proposito, un suo lavoro "Sulla polarizzazione della luce diffusa del cielo", pubblicato sull' Annuario meteorologico ", a cur"a del Comitato direttivo della Societa meteorologica italiana (Anno IV. 1889).

Giuseppe Pisati (Pavia 1842 - Roma 1901), fu instancabile ricercatore, di una onesta professionale e di uno scrupolo eccezionali, forse eccessivi. Nel 1872 fu nominato professore di fisica sperimentale nella Universita di Palermo e nel 1878 fu chiamato all'insegnamento della fisica tecnica nella Scuola d'ingegneria di Roma. Esula dal nostro compito laccennare alle sue accuratissime ricerche di chimica-fisica, di lisica 
tecnica, dell'elasticita e della tenacita dei metalli a diverse temperature, ecc. Non possiamo non accennare però al poderoso lavoro, intrapreso assieme a Pucci sullo studio della gravita e sulla determinazione del valore di $g$, condotto con tanta varieta di metodo e di condizioni, con tanto acume e precisione, da potersi ben citare come esempio, non facilmente superabile, di perseveranza illuminata e di serieta d'intenti. Per quanto concerne la geofisica, possiamo ricordare la sua partecipazine alle campagne talassografiche promosse nel 1883 e nel 1884 dall'Ufficio idrografico della Marina; una nel Mediterraneo occidentale, l'altra nel Mediterraneo orientale. Studio con impegno l'abbondante materiale raccolto. Con particolare riferimento alla densita dell'acqua marina, egli ideò e realizzò apprezzabili innovazioni nei metodi e negli strumenti di misura, sopra tutto allo scopo di conciliare la precisione - alla quale egli sommamente aspirava - con la speditezza delle misure e con le difficili condizioni della navigazione.

Un altro valente cultore di geofisica dell'Ottocento fu GIULro Grablovitz (Trieste 1846 - Casamicciola 1928). Egli fondo l'Osservatorio geofisico di Ischia (Grande Sentinella) nel 1886, e lo dotò di apparecchi sismici di sua ideazione. Fra i vari accorgimenti introdotti da Grablovitz nella registrazione dei fenomeni sismici, merita particolare menzione il sistema d'astatizzazione della componente verticale del movimento (1889). La molla a spirale, che ad un estremo regge la massa stazionaria, e inclinata di $45^{\circ}$ sulla verticale ed e connessa a detto estremo con un'asta, ad essa ortogonale, ruotante attorno ad un fulcro. Il principio d'astatizzazione $\dot{e}$ il seguente: le dimensioni del sistema oscillante possono essere scelte in modo che, quando la massa si innalza o si abbassa, con l'accorciamento o l'allungamento della molla, la diminuzione o l'aumento del momento della forza della molla vengono sempre compensati, in prima approssimazione dalla diminuzione o dall'aumento del momento del peso. Tale principio di astatizzazione e ancora oggi adottato dalle migliori fabbriche di sismografi.

Si interesso di altre questioni di geofisica, con speciale riguardo allo studio fisico delle acque termali, delle fumarole, sabbie calde ed emanazioni in genere, alla meteorologia e all'astronomia. La mareografia attrasse costantemente la sua attenzione. Fu tra $\mathrm{i}$ primi ad interessarsi delle maree del Mediterraneo, di cui studio le isorachie (1891). Altri lavori riguardano le maree d'Ischia (1890), le maree dell'Adriatico (1892), un nuovo modello di mareografo (1900), ecc.

Pioniere di larga notorieta nelle ricerche sismologiche e vulcanologiche fu Giuseppe Mercalli (Milano 1850 - Napoli 1914). Dopo la laurea 
in scienze naturali (1874), si interesso dello studio dei terreni glaciali della Lombardia. La sua attenzione fu successivamente attratta dai fenomeni sismici e vulcanici, ai quali dedicò intera la sua attivita. Pubblicò memorie sui terremoti di Casamicciola (1883), isole Pontine (1892), di Messina (1908), ecc. Tracciò la prima carta sismica d'Italia. Il suo nome e sopra tutto legato ad una scala sismica empirica, ancora universalmente accettata - sia pure con modificazioni - per la valutazione approssimativa dell'intensita di un terremoto.

Lasciò pure apprezzate pubblicazioni sui vulcani spenti ed attivi, con particolare riguardo a quelli italiani. Di esse ricorderemo "I vulcani attivi della Terra ", "Osservazioni petrografiche e geologiche sui vulcani " (1889).

Adolfo Bartolu (Firenze 1851 - Pavia 1896), insegno fisica e fisica matematica in diverse universita italiane (Sassari, Firenze, Catania, Pavia). Compì ricerche in diversi rami della fisica e della chimica-fisica, con particolare riguardo all'irraggiamento, all'ottica, all'elettrochimica e alla calorimetria. Gli ultimi anni della sua vita li dedico alla misura del calore solare. Tale lavoro fu infatti da lui iniziato nel 1885 e continuato senza interruzione fino al 1896. Bartoli, in diverse localita, raccolse migliaia di osservazioni, allo scopo di studiare le leggi e le cause della variazione del calore solare nel tempo.

Si dedicò anche alla misura della temperatura della lava eruttata dall'Etna, in una grande eruzione di quell'epoca.

Nell'ambito degli studi sismologici, risulta di primaria importanza l'opera di Adolfo Candani (Roma 1856-1904). Lavoro presso l'Osservatorio geodinamico di Rocca di Papa e nel $R$. Ufficio centrale di meteorologia e geodinamica. Ideò numerosi apparecchi per la segnalazione e la registrazione dei terremoti. La teoria dell'elasticità prevede, come è noto, l'insorgere di due sistemi di onde - longitudinali e trasversali - in un corpo solido sollecitato: estendendo tali concetti alla Terra, Cancani sostenne che tali sistemi di onde dovevano venir prorocati anche dai terremoti, avviando cosi a soluzione il problema fondamentale di poter risalire alla determinazione delle coordinate ipocentrali, sulla base della registrazione dei treni d'onda longitudinale e trasversale. Fra gli altri suoi meriti, ha quello di aver formulato per primo (1903) una relazione fra i gradi della seala empirica dell'intensita dei terremoti e le corrispondenti accelerazioni, dando cosi l'avvio ad una serie di ricerche sull'argomento, alla quale porto successivamente contributi anche Gutenberg.

In campi piu vasti si estese l'impegno di un altro valoroso ricercatore, Luigr De Mireirt (Milano 1857 - Padova 1936), letterato, geografo e geofisico. Limitero le mie considerazioni a questo suo ultimo aspetto. 
Fece dapprima pregevoli indagini di meteorologia, considerata come un'estensione della dinamica dei fluidi (1890). I principi che regolano la distribuzione della temepratura sulla superficie terrestre sono esposti nell'opera sulle cause dell'era glaciale (1895), di cui diremo piu avanti. Cospicui contributi porto pure negli studi mareografici, indagando sulla propagazione dell'onda di marea nella laguna veneta, considerata come. rete di canali (1.905), sula distribuzione della salsedine e della temperatura in mare (1912), sulle onde interne e sulla propagazione di marea nell'alto Adriatico (1916), ecc. Si interessò pure di questioni inerenti alla teoria dell'elasticita.

Nel 1902 pubblico il Trattato di geografia fisica, prima pregevole opera italiana in questa materia.

Ma Luigi De Marchi fu pioniere sopra tutto nel campo della glaciologia. Nel secolo scorso, una volta dimostrato che le grandi invasioni glaciali quaternarie non erano dovute a espansioni di una calotta glaciale polare, bensì ad estensioni di ghiacciai montani, parve naturale riferire tali espansioni ad aumento generale delle precipitazioni acquee, quindi delle nevi in montagna: in altri termini, il fenomeno glaciale (a quell'epoca, si parlava generalmente, di una sola glaciazione - "monoglacialismo "), doveva essere stato preparato da un periodo climatologico caldoumido. Piu accurate indagini provarono pero che gli avanzamenti glaciali corrispondevano a gruppi d'anni freddo-umidi, mentre le contrazioni conseguivano a gruppi d'anni caldo-ascintti.

Nel 1895 ("Sui climi terrestri durante l'era glaciale e quaternaria e sulla cause che hanno contribuito a modificarli ") De Marchi provo che il bilancio di un ghiacciaio e regolato piu dall'ablazione estiva che dalla precipitazione nevosa invernale. Ne conseguiva che anche i grandi periodi del glaciale quaternario erano dovuti, come le oscillazioni secolari dei ghiacciai attuali, a lunghi periodi di clima freddo-umido, specialmente a estati fredde. L'espansione glaciale va quindi imputata a un abbassamento di temperatura più che ad un aumento di precipitazione. E noto che, fra le cause astronomiche delle glaciazioni, sono invocate le variazioni secolari di luminosita delle varie categorie di stelle, e in particolare le variazioni repentine e transitorie alle quali sarebbero soggette tutte le stelle, compreso il Sole. Secondo Zinner, repentini aumenti e successive variazioni della radiazione solare, darebbero come effetti postumi, le ere glaciali. Altri invocano, forse a maggior ragione, variazioni della. radiazione solare come conseguenza di variazioni della eccentricita dell'orbita terrestre, combinata con la precessione degli equinozi e l'inclinazione dell'asse terrestre. 
De Marchi dimostro fin dal 1895 (Le cause dell'Era Glaciale) che le rariazioni climatiche, connesse alle glaciazioni, possono spiegarsi anche con variazioni della trasparenza atmosferica. Secondo tale ipotesi, il fenomeno glaciale poteva attribuirsi ad un intorbidamento dell'atmosfera, comunque determinato: ad una diminuzione, anche non molto rilevante, del coefficiente di trasparenza, risponderebbe un abbassamento di parecchi gradi nella temperatura annua, ed una diminuzione dell'escursione annua, e quindi della temperatura estiva.

Tale ipotesi conserva, ancor oggi, piena attendibilita.

Fra i pionieri della sismologia, uno dei piu laboriosi fu senza dubbio Giovanni Agamennone (Rieti 18508 - Roma 1949). Si laureò in fisica a Roma (1884), fu assistente di sismologia all'Osservatorio di Ischia, prima, quindi presso l'Ufficio Centrale di meteorologia e geofisica di Roma, dove fece ritorno nel 1929 come direttore del servizio sismico, dopo aver diretto dal 1899 l'Osservatorio di Rocea di Papa. Nel biennio 1895-'97 fu chiamato in Turchia, ad organizzarvi il servizio sismico. Fu fra i membri fondatori della Societa sismologica italiana (1895). Fra i vari riconoscimenti nazionali ed esteri, fu nominato professore onorario dell'Universita di Atene.

Ideo diversi tipi di sismoscopi e di sismografi, che trovarono larga diffusione in Italia e all'estero. Pubblicó circa trecento lavori su argomenti vari di sismologia, con particolare riguardo alla sismografia, alle osservazioni macrosismiche e alla determinazione delle profondita ipocentrali, che egli riteneva di piccolo valore $(15-20 \mathrm{~km}$ al massimo): fu infatti avversario irriducibile della possibilità di movimenti sismici profondi (successivamente provati fino a profondita di $750 \mathrm{~km}$ circa).

Sebbene Gruseppe Vicentini (1860-1944), professore di fisica sperimentale nell'Universita di Padova, abbia svolto la sua attivita sopra tutto come fisico, egli puo essere ritenuto uno dei piu illustri pionieri nella sismologia strumentale. I sismografi a registrazione meccanica da Iui ideati, verso la fine del secolo scorso, si diffusero rapidamente in tutto il mondo e furono - per circa un trentennio - fra i piu apprezzati.

E veniamo ora ad uno dei massimi cultori di fisica-matematica che abbia avuto l'Italia nell'ultimo secolo: CARLo Somgliaxa (1860-195̃5). Insegnò dapprima nell'Universita di Pavia (1892-1903); passò quindi a Torino, dove tenne la cattedra di fisica matematica fino al suo collocamento a riposo (1935).

Tra i pregevolissimi suoi lavori sulla teoria dell'elasticita, ci limiteremo a citare: "Sopra gli integrali delle equazioni della isotropia elastica " (in 'Nuovo cimento', 1885), in cui stabilisce alcune formule fon- 
damentali, che vanno sotto il suo nome e che riducono il problema generale dell'equilibrio di un corpo elastico alla ricerca di soluzioni per particolari condizioni al contorno. Fra le ricerche di Somigliana nel campo della geodesia o della geofisica fondamentale e la sua "Teoria generale el dcampo gravitazionale dell'ellissoide di rotazione " (in Mem. della Soc. Astr. Ital., 1929).

In sismologia rivestono particolare importanza i suoi lavori a Sulla propagazione delle onde sismiche " (Rend. Lincei, 1907), in cui vengono ritrovate, per altra via, le onde superficiali di Rayleigh. Somigliana prova che tali onde possono nascere dall'accoppiamento di un'onda longitudinale e di un'onda trasversale piane, caratterizzate da identiche velocita di propagazione superficiali. Le condizioni a cui queste onde sovrapposte devono soddisfare in superficie, conducono Somigliana all'equazione

$$
16\left(1-\frac{V_{R}^{2}}{v_{1}^{2}}\right)\left(1-\frac{V_{R}^{2}}{v_{2}^{2}}\right)=\left(2-\frac{V_{R}^{2}}{v_{2}^{2}}\right)^{4},
$$

$\left[V_{R_{1}}=\right.$ velocita onde superficiali; $\quad v_{1}=$ velocita onde longitudinali $\mathrm{e}$ $v_{2}=$ velocita onde trasversali].

che coincide con la classica equazione delle onde di Rayleigh. Dal punto di vista geofisico, esiste però una sostanziale differenza fra le due teorie. Nella classica teoria di Rayleigh, infatti, solo la radice minore dell'unita dell'equazione riportata ha significato fisico. Nella teoria di Somigliana non esiste tale limitazione: sicche in essa tutte e tre le soluzioni dell'equazione di condizione soddisfano al problema. Pertanto, tre sistemi di onde, e non uno soltanto, possono nascere in superficie, quando le coppie di onde sovrapposte considerate soddisfano alle condizioni di partenza. Inoltre, mentre nell'originaria teoria di Rayleigh, il rapporto della componente verticale del movimento alla componete orizzontale nella direzione di propagazione, risulta ben determinato - e quindi espresso da un numero fisso, che generalmente non concorda con le osservazioni nella teoria di Somigliana tale rapporto può avere qualsiasi valore.

La teoria di Somigliana conduce quindi ad una generalizzazione della soluzione trovata da Rayleigh per la propagazione delle onde piane alla superficie di un mezzo piano, omogeneo, isotropo, illimitato.

Sotto certi aspetti, anche Luigr PaLazzo (1861-1933) può essere citato fra i pionieri della geofisica in Italia, specie per ciò che si riferisce al magnetismo terrestre (costrui carte isodinamiche ed isocline per l'Italia e colonie) e per l'organizzazione della rete meteorologica italiana. Lo è senz'altro, particolarmente nei riguardi della sismologia, EMmLio 
OdDone (1864-1910). La sua attivita si estese a parechi rami della geofisica. Collaboro con Sella (v.) in alcune ricerche di magnetismo terrestre. Ma come si e detto, egli sopratutto si interesso di questioni di sismologia. Fra i molti lavori compiuti in questo campo (sulla determinazione delle profondita ipocentrali, sulla propagazione delle onde sismiche spaziali e superficiali, ecc.), merita particolare menzione un metodo originalissimo sulla determinazione del modulo di Young delle rocce ("Determinazione dinamiche del modulo di elasticità di Young delle rocce " - Annali dell'Uff. Centr. di Meteorologia e Geodin., XXXIII, 1911, Parte 1a). Tale metodo si sintetizza nella formula

$$
\frac{1}{E}+\frac{1}{\tilde{E}_{1}}=\frac{16 a^{5}}{13,5 m 2 g H R^{2}},
$$

dove $E$ e il modulo di Young dell'acciaio, di cui e composta una biglia di raggio $R$ e di massa $m$; $H$ l'altezza da cui si lascia cadere la biglia ed $a$ il raggio della traccia circolare che la biglia stessa lascia sulla superficie levigata, cosparsa di nerofumo, del campione di roccia, di cui si vuol determinare il modulo $E_{1} ; g$ e l'accelerazione di gravita.

Oddone pervenne a questa formula partendo dalla teoria matematica degli urti, sviluppata da Love e, piu compiutamente, da Hertz.

Fu presidente dell'Associazione internazionale di sismologia per il triennio 1933-1936.

Citeremo ancora ALFonso Sella (Biella 1865 - Roma 1906), figlio del grande statista e mineralogista Quintino Sella. Insegno fisica complementare all'Universita di Roma.

Nel suo insegnamento propugno la necessita di una buona cultura matematica nelle indagini fisiche. Ise sue notevoli ricerche nel campo fisico sono tutte improntate alla riprova di questa necessita: l'algoritmo matematico come ausilio potente ed insostituibile nel conseguimento della realta fisica. Esula dal mio assunto di riferire sui risultati di Alfonso Sella nelle sue ricerche di fisica, che vertono principalmente sui raggi $\mathrm{X}$, sulla propagazione della luce nei cristalli magnetici, sulla reciprocità fra fenomeni ottico-magnetici e magneto-ottici, sui fenomeni di ionizzazione dei gas, ece.

Sotto l'influenza del suo amico Prof. Keller, Sella dedicò parte della sua attivita anche alla geofisica e specialmente al magnetismo terrestre. In collaborazione con Emilio Oddone studio le rocce magnetiche delle Alpi centrali, trovando numerosi punti distinti (anomali) e accertando che la distribuzione del magnetismo non e in relazione alcuna con la direzione del campo terrestre. 
Da quella serie di ricerche vennero altre notevoli deduzioni. Gia Nacedonio Melloni avera provato che roce, portate a intenso calore e poi fatte raffreddare lentamente, diventano magnetiche sotto l'azione del magnetismo terrestre. Sella e Oddone ne deducono che una roccia eruttiva presenta quella distribuzione magnetica che le fu comunicata dal campo terrestre nell'atto del raffreddamento (Rend. Acc. Lincei, 1891). Di qui la possibilità, dal punto di vista geologico, di dedurre dall'orientazione attuale della distribuzione piegamenti, sollevamenti, torsioni, ecc. avvenute nella roccia; e, dal punto di vista geofisico, di trarre nozioni sulla direzione del campo terrestre all'epoca del raffreddamento della roccia, la ove si possa ammettere che la roccia sia rimasta ferma in posto. Sono i primi passi del paleomagnetismo.

Nel 1891 il matematico tedesco Keller era impegnato in un esame critico dei metodi per determinazione della densita media della Terra. Dai colloqui avuti con Keller, maturo in Alfonso Sella il proposito di studiare la questione del corpo di massima attrazione su un punto o secondo una direzione. Tale proposito si concreto in due pregevolissimi lavori teorici, apparsi fra i Rendiconti dell'Accademia dei Lincei negli anni 1891 e 1893: essi si concretarono nella discussione sulla forma piu opportuna da darsi al corpo attraente nella misura della densita media della Terra (1893). Sempre fra i Rendiconti dell'Accademia predetta, Sella pubblico i risultati di una serie di misure relative della componente orizzontale del magnetismo terrestre sul Monte Rosa, a Biella e a Roma.

Va ancora ricordato che, occupandosi di raclioattivita, Sella scoprì per primo la presenza di emanazioni radioattive nelle acque potabili comuni (Rend. Acc. Lincei, 1902). Determinò inoltre (Rend. Acc. Lincei, 1904) la radioattivita delle pozzolane dei dintorni di Roma, fissandola intorno alle 0,001 unita uraniche.

Parecchi altri uomini di scienza, nati nella seconda meta dell'ottocento, hanno svolto, almeno in parte, attivita pionieristiche nell'ambito della geofisica: Antonino Lo Surdo (1880-1949), Francesco Vercelli (1883-1952), Mario Texani (1886-1955). . . Prevale peró in essi la veste di sistematori sulla traccia di sentieri preesistenti e gia ben delineati. In ogni modo non posso chiudere senza citare Domenico Pacini (Marino 1878 - Roma 1934), scopritore della radiazione cosmica. Eseguì numerosi lavori di fisica terrestre; particolarmente importanti quelli sulla radiazione penetrante e quelli sull'azzurro del cielo. Lo studio sull'azzurro del cielo lo condusse ad un metodo per la verifica del numero di Avogadro, con conferma dei risultati ottenuti per altre vie. Il suo nome pero è sopra tutto legato alle ricerche sull'energia penetrante, di cui dimostrò 
l'origine cosmica con una serie di esperienze eseguite nel lago di Albano che, provocando l'attenuazione con la profondita, provavano l'origine extra terrestre della radiazione suddetta. Gia nel 1910, dopo una serir di misure eseguite contemporaneamente su mare e su terra ferma, trovava che il numero di ioni dovuti alla radiazione penetrante sul mare era circa i $2 / 3$ di quello osservato su terra ferma. Da una critica delle probabili cause di un così alto valore della ionizzazione della libera atmosfera alla superficie del mare, poteva concludere che "una parte non piccola della radiazione penetrante presente nell'aria, e in modo particolare quella parte che $\dot{e}$ soggetta ad oscillazioni anche notevoli, ha origine indipendente dall'azione diretta delle sostanze attive contenute negli strati su. periori della crosta terrestre " ( La radiazione penetrante sul mare». Ann. Uff. Centr. Met. e Geod., 1910). 